\title{
A Data Bootstrapping Recipe for Low-Resource Multilingual Relation Classification
}

\author{
Arijit Nag \\ IIT Kharagpur \\ arijitnag@iitkgp.ac.in \\ Niloy Ganguly \\ IIT Kharagpur, Leibniz University Hannover \\ niloy@cse.iitkgp.ac.in
}

\begin{abstract}
Relation classification (sometimes called 'extraction') requires trustworthy datasets for finetuning large language models, as well as for evaluation. Data collection is challenging for Indian languages, because they are syntactically and morphologically diverse, as well as different from resource-rich languages like English. Despite recent interest in deep generative models for Indian languages, relation classification is still not well-served by public data sets. In response, we present IndoRE, a dataset with $21 \mathrm{~K}$ entity- and relation-tagged gold sentences in three Indian languages, plus English. We start with a multilingual BERT (mBERT) based system that captures entity span positions and type information, and provides competitive monolingual relation classification. Using this system, we explore and compare transfer mechanisms between languages. In particular, we study the accuracyefficiency tradeoff between expensive gold instances vs. translated and aligned 'silver' instances. We release the dataset for future research. ${ }^{1}$
\end{abstract}

\section{Introduction}

Relation classification (sometimes called relation 'extraction' or RE) is the task of identifying a semantic relation (from a catalog of canonical relations) that holds between two nominal entities in text. Related to semantic role labeling, it is a critical capability for question answering and knowledge graph (KG) augmentation. The key challenge in RE is the diversity of textual expressions of canonical relations. WikiData, among the largest public KGs, has a hundred million entities with aliases but only thousands of wellinstantiated canonical relations, with relatively sparse textual descriptions of relations, particularly in low-resource languages.

\footnotetext{
${ }^{1}$ https://github.com/NLPatCNERG/IndoRE
}

\author{
Soumen Chakrabarti \\ IIT Bombay \\ soumen@cse.iitb.ac.in
}

Animesh Mukherjee

IIT Kharagpur

animeshm@cse.iitkgp.ac.in

Low-resource languages, including many Indian languages, neither have well-tuned standard NLP pipelines, nor unsupervised corpora as large as resource-rich languages. Not only are they often morphologically distant from resource-rich language families, but even their syntax for relation expression differs. E.g., while subject-predicateobject (infix) is more common in English, subjectobject-predicate (postfix) is more common in Indic languages: "Ravi went to Delhi" (En) vs. रवि दिल्ली गया था/“Ravi Delhi went-did" (Hi). Word order is more relaxed: दिल्नी गया था रवि/“Delhi went-did Ravi" is acceptable; only the entity types help orient the predicate. Verb particles are more common than in English: "Talk to him" (En) becomes उससे बात करो/ "him-to talk do" (Hi) or তার সাথে কথা বলুন/“him with speech talk” (Bn). Transliteration and code switching are rampant: "I called him yesterday" (En) translates to " मैंने कल उसे कॉल किया था"/“I yesterday him call did-past", where call is transliterated.

Against this backdrop of diverse textual expression of relational facts, collecting high-quality labeled data for Indian language RE is a major challenge, particularly given data-hungry state-of-theart neural RE systems. Responding to these pressures, we contribute a multilingual RE testbed for low-resource Indian languages. Specifically, we build and contribute IndoRE, a diverse and rich set of entity- and relation-annotated sentences in three Indian languages - Bengali (Bn), Hindi ( $\mathrm{Hi}$ ) and Telugu (Te). In addition, we provide labeled English (En) RE instances. Our motive is to study and catalog a diversity of protocols for transferring RE capability across languages, chiefly in the resource-rich to resource-poor direction, starting from the "each language for itself" (ELFI) operating point. Owing to distributional fidelity, we generally expect that, with 'sufficient' gold training data, ELFI will show strong performance; the catch being the expense of collecting gold in- 
stances in resource-poor languages.

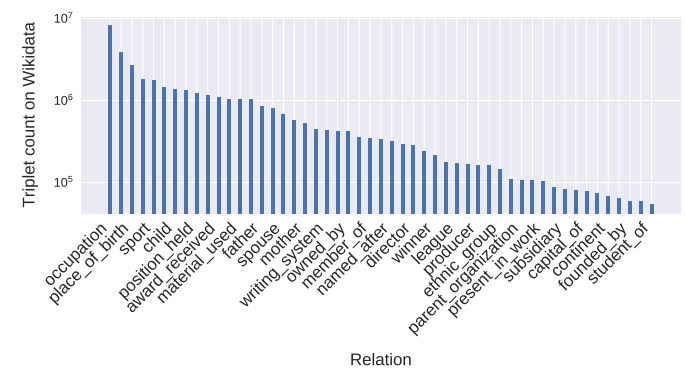

Figure 1: Number of KG triples involving the selected relations.

To investigate the extent to which various modes of transfer can compensate for gold instances, we start by designing a competitive base RE system built on mBERT. ${ }^{2}$ By sharing a multilingual wordpiece vocabulary and training on a vast multilingual corpus, mBERT already lends some support to go beyond ELFI. Beyond this baseline, we explore ways to train models for RE in a target language by 'borrowing' training instances from a given source language, translating and aligning, and preparing 'silver' instances to train an RE system for the target language. Such model transfer (Kozhevnikov and Titov, 2014) may be attempted in a zero-shot mode, where no gold-labeled instances are available in the target language, or in a few-shot mode where a few such instances are prepared at some cost. We also explore an instance transfer setting, in which we train the RE module using only English instances, and translate target language test instances to English as well.

\begin{tabular}{|c|c|}
\hline Relation $(r)$ : & spouse \\
\hline Sentence $(s)$ : & $\begin{array}{l}\text { विराट कोहली और अनुष्का शर्मा ने } 2017 \text { में इटली } \\
\text { में शादी कर ली थी }\end{array}$ \\
\hline English: & $\begin{array}{l}\text { Virat Kohli and Anushka Sharma got married } \\
\text { in Italy in } 2017 .\end{array}$ \\
\hline Entity pair: & विराट कोहली , अनुष्का शर्मा \\
\hline Lexical distance: & 1 \\
\hline Relation $(r)$ : & award_received \\
\hline Sentence $(s)$ : & $\begin{array}{l}\text { बृहत-पिंगला गुजराती भाषा के विख्यात साहित्यकार } \\
\text { रामनारायण पाठक द्वारा रचित एक छंद शास्त्र है जिसके } \\
\text { लिये उन्हें सन् } 1956 \text { में गुजराती भाषा के लिए मरणो- } \\
\text { परांत साहित्य अकादमी पुरस्कार से सम्मानित किया } \\
\text { गया . }\end{array}$ \\
\hline English: & $\begin{array}{l}\text { Brihat-Pingala is a verses scripture composed } \\
\text { by noted Gujarati litterateur Ramnarayan } \\
\text { Pathak for which he was posthumously } \\
\text { awarded the Sahitya Akademi Award for } \\
\text { Gujarati language in } 1956 \text {. }\end{array}$ \\
\hline Entity pair: & बहत-पिंगल , साहित्य अकादमी परस्कार \\
\hline Lexical distance: & 24 \\
\hline
\end{tabular}

Table 1: Sample 'simple' and 'complex' relations.

\footnotetext{
${ }^{2}$ https://github.com/google-research/bert/ $\mathrm{blob} / \mathrm{master} / \mathrm{multilingual}$.md
}

The presence of multiple languages, with diverse levels of similarity between them, brings a new dimension to our study. We find that 0 -shot model transfer works better between Indian languages than from English to an Indian language, but is still behind ELFI by $11.27 \%$ (Bn), $11.06 \%$ (Hi) and $13.43 \%(\mathrm{Te})$ macro F1. Additional finetuning with 10-shot target gold instances reduces the gap to $3.76 \%(\mathrm{Bn}), 2.8 \%(\mathrm{Hi})$ and $4.23 \%(\mathrm{Te})$. Effectiveness of model transfer also shows interesting and intuitive patterns and variations across language pairs in our transfer framework, TransRel. We observe that translating test instances to English helps a model trained on English instances perform well; however, the gain diminishes as we add a few training samples translated to English from the target language.

\section{IndoRE data collection process}

We focus on three Indian languages: Hindi (Hi), Bengali (Bn) and Telugu (Te). Our intention was to pick two languages ( $\mathrm{Hi}$ and $\mathrm{Bn}$ ) that are more similar to each other than to the third language, Te. However, each of these languages has certain distinctive features not found in the others. As an example of a resource-rich language, we include English (En).

In this section, we first discuss the limitations of existing multilingual RE data sets. Then we describe the data collection process, starting with relation selection, and followed by evidence sentence collection, data processing and automatic annotation. Then we discuss annotation evaluation and cleanup using human annotators.

\subsection{Available datasets and limitations}

Several groups (Hendrickx et al., 2010; Han et al., 2018; Riedel et al., 2010; Zhang et al., 2017) have released distant-supervised or humanannotated RE datasets in English. As we shall argue, translating them to respective Indian languages may not generate a good representative collection. The human-curated supervised datasets SemEval'10 (Hendrickx et al., 2010) and FewRel (Han et al., 2018) contained $\sim 10 \mathrm{k}$ and $56 \mathrm{k}$ sentences respectively over nine and 80 relations. The relations for FewRel are from Wikipedia. As the relations mainly focus on an English corpus, most of them do not have sufficiently many representative candidates in Indian languages as they are not relevant to the Indian context. E.g., FewRel 
has a relation taxon_rank with sample passage "...more formally brought together the three families, Agapanthaceae, Alliaceae, Amaryllidaceae, under the single Asparagalean monophyletic family, now renamed Amaryllidaceae from Alliaceae, reversing the Dahlgrenian process of family splitting...". This relation has no candidate in any Indian language.

The distant-supervised dataset TACRED (Zhang et al., 2017) released relations which are not from existing knowledge-bases like Wikipedia or DBpedia. TACRED was created based on query entities and annotated system responses in the yearly TAC KBP evaluations. Therefore, this data is very distinct and specific to the TAC KBP corpus, resulting in certain cultural gaps. For example "Billy Mays, ... pop culture icon, died at home in Tampa." is evidence for the relation city_of_death between entities "Billy Mays" and "Tampa". In Bengali, মারা যান means 'died' for commonors, but for celebrities, শেষ নিঃশ্বাস ত্যাগ করেন (last breath release did) is more common. These cultural variations cannot be captured by translating sentences very specific to En.

The NYT data set (Riedel et al., 2010) includes 42 relations from Freebase, but entity types are restricted to only businesses, people and locations, which makes it restrictive and less diverse. We provide a comparison among the datasets in Table 2.

\begin{tabular}{l|c|c|c|c}
\hline Dataset & Language & KB & \#Relation & \#Sentence \\
\hline SemEval'10 & En & - & 9 & 10,717 \\
\hline FewRel & En & Wikidata & 80 & 56,000 \\
\hline TACRED & En & TAC KBP & 42 & 119,474 \\
\hline NYT & En & Freebase & 53 & 126,184 \\
\hline IndoRE & En,Bn,Hi,Te & Wikidata & 51 & 32,610 \\
\hline
\end{tabular}

Table 2: Comparison of different datasets.

\subsection{Relation selection}

We select 51 relations from WikiData using stratified sampling, proportional to the number of available (subject, relation, object) triples per relation, including all languages present in WikiData. Figure 1 shows triplet counts in decreasing order for various relations. Some relations are (linguistically) easy to express, e.g., spouse, whereas other relations are complex and need longer sentences to express, e.g., award_received; see Table 1. One rough indicator of the complexity of a relation is the number of tokens (lexical distance) between the two related entities. For various relations, the average lexical distance in different languages is shown in Appendix A, Figure 3.

\subsection{Evidence sentence collection}

After selecting the relations, we collect potential evidence sentences for each relation in each language. As there is no direct way to obtain large numbers of gold evidence sentences, we apply distant supervision (Mintz et al., 2009).

We sample a set of entity pairs for each relation in each language using stratified sampling proportional to the sentence count per entity pair. We collect a potentially noisy set of evidences per entity tuple, i.e., per $\left(e_{1}, e_{2}\right)$, by collecting a maximum of 1000 sentences, based on keyword search.

The entire English, Bengali, Hindi and Telugu Wikipedia dumps ${ }^{3}$ are processed using Pyserini ${ }^{4}$, an open-source information retrieval toolkit. We query its index with entity pair $\left(e_{1}, e_{2}\right)$ separated by space, and collect the top- 1000 results. The intuition of such distant supervision is that if two entities participate in a relation, any sentence which mentions these two entities has some chance of expressing it. To enforce data diversity, we further collect sentences from mixed sources, by querying the Google Web search engine API with $\left(e_{1}, e_{2}\right)$ and selecting the top five response URLs, ${ }^{5}$ which are fetched. From the HTML, we extract sentences where both the entities are present.

\subsection{Data filtering and annotation}

Distant supervised methods can yield noisy sentences, i.e., even though they contain the desired entity pair $\left(e_{1}, e_{2}\right)$, they may not provide evidence of the relation $r$. We filter out some non-evidence sentences as follows. We generate an embedding $\left(e m b_{s}\right)$ of each collected sentence - after removing the two entity mention spans - as an average word embedding using mBERT. Similarly, we obtain relation embedding $\left(e m b_{r}\right)$ as an mBERT embedding of the relation by taking average over all the individual contextual embedding of the description and alias of a particular relation from WikiData. Given a sentence $s$ and corresponding desired relation $r$, we retain sentences for which $\cos \left(e m b_{s}, e m b_{r}\right) \geq \tau$, where $\tau$ is a threshold which is empirically determined for each language (typically, 0.3 gave the best results).

Next we mark the entity spans in each sentence by two special marker pairs $\left[E_{1}\right]\left[/ E_{1}\right]$ and

\footnotetext{
${ }^{3}$ https://dumps .wikimedia.org/

${ }^{4}$ https://github.com/castorini/pyserini/

${ }^{5}$ https://pypi.org/project/google/
} 


\begin{tabular}{ccc}
\hline Language & \#Sentence & \#Distinct entity pair \\
\hline $\mathrm{En}$ & 8486 & 7232 \\
$\mathrm{Bn}$ & 8291 & 5321 \\
$\mathrm{Hi}$ & 6979 & 4399 \\
$\mathrm{Te}$ & 8854 & 4041 \\
\hline Total & 32610 & 20993 \\
\hline
\end{tabular}

Table 3: Salient statistics of IndoRE.

$\left[E_{2}\right]\left[/ E_{2}\right]$ as it is useful to learn a generalized embedding for entities which can help in relation extraction (Soares et al., 2019). For English sentences, we obtain entity types using the spaCy NER tagger. ${ }^{6}$ For Indian languages we translate the entity into English and obtain the entity type with the same tool.

\subsection{Annotation evaluation}

After applying the preprocessing steps described above, we clean the instances by employing human annotators. We assign instances to annotators who are fluent in the language of the instance. We provide the canonical relation name $(r)$, a sentence $s$ marked with entity pair spans, entity pair $\left(e_{1}, e_{2}\right)$ and entity types $\left(e t_{1}, e t_{2}\right)$. A sample is shown (with En translation added).

\begin{tabular}{l|l}
\hline Relation $(r):$ & spouse \\
Sentence $(s):$ & {$\left[E_{1}\right]$ मिशेल ओबामा $\left[/ E_{1}\right]$ अमेरिका की पूर्व राष्ट्रपति } \\
& {$\left[E_{2}\right]$ बराक ओबामा $\left[/ E_{2}\right]$ की पत्नी हैं , } \\
& एवं अमेरिका की प्रथम महिला रह चुकी हैं \\
\hline English & $\begin{array}{l}\text { Michelle Obama is the former first lady } \\
\text { and wife of former American president } \\
\text { translation: } \\
\text { Barack Obama }\end{array}$ \\
\hline Entity pair: & $\begin{array}{l}\text { मिशेल ओबामा, बराक ओबामा } \\
\text { Entity type pair: }\end{array}$ \\
\hline
\end{tabular}

We also provide a list of entity types and necessary context by providing a relevant link to the page where the relation definition and other related information are explained. The annotators are asked to perform the following tasks.

(a) Discard the sentence if it does not entail relation $r$ between $e_{1}$ and $e_{2}$.

(b) Correct the entity spans $\left[E_{1}\right] \cdots\left[/ E_{1}\right]$ and $\left[E_{2}\right] \cdots\left[/ E_{2}\right]$ if necessary.

(c) Correct the entity types et $t_{1}$ and et $_{2}$ consulting the list of entity types, if needed.

We first ran a pilot study with 100 sentences for each language and found that the inter-annotator agreement was more than $85 \%$. Thereafter, we processed the rest of the data with one annotator per instance. Statistics of the final cleaned data are provided in Table 3.

\subsection{Silver instance generation}

To increase coverage of examples for the relations located at the tail of the distribution as reported in

\footnotetext{
${ }^{6}$ https://spacy.io/
}

Figure 1, we also generate (semi-) synthetic or 'silver' data that is used for model transfer from one language to another, commonly from a resourcerich to low-resource language. We illustrate the process using En and Hi.

We translate gold-labeled En instances into Indian languages (say, Hi) using the Google translate API. ${ }^{7}$ As there is no commonly available NER tagger in Indian languages, we use English entities to mark the entity span in Indian languages. We translate the entities of the source English sentence into Hi and mark the entity spans in the target synthetic sentence using a simple heuristic described in Appendix B. Finally, we borrow the entity types from the source sentence. The following example walks through this process in detail. As we do not rectify the entity mapping using human annotators, we term this set as silver data.

\begin{tabular}{l|l}
\hline English & $\begin{array}{l}\text { Virat Kohli and Anushka Sharma got married } \\
\text { in Italy in 2017. }\end{array}$ \\
\hline Entity (Type) & Virat Kohli (Person), Anushka Sharma (Person) \\
\hline Translated Hindi & $\begin{array}{l}\text { विराट कोहली और अनुष्का शर्मा ने } 2017 \text { में इटली में } \\
\text { शादी कर ली थी }\end{array}$ \\
\hline Entity mapping & विराट कोहली (Person), अनुष्का शर्मा (Person) \\
\hline
\end{tabular}

\section{Base RE module}

We build a base RE module which will be used for $\mathrm{RE}$ in Indian languages with various cross lingual transfer schemes. As depicted in Figure 2, our architecture is built on multilingual BERT (mBERT), a masked language model. ${ }^{8}$

For effective relation extraction in a multilingual setting, at the input layer, we provide entity tagged inputs; the summarization layer collects important information about the sentence and entities; finally, the relation extraction layer predicts the relation using entity type information along with other contextual information. We try different variations of the architecture and describe the best preforming variation here. We will describe some important details of preparing the input, the summarization, and the output RE layers.

\subsection{Input layer}

We insert special marker tokens in the input sentence $(s)$ for effective presentation to mBERT. We mark entity spans by special tokens $E_{1}$ and $E_{2}$ as

\footnotetext{
${ }^{7}$ https://cloud.google.com/translate

${ }^{8}$ https://github.com/google-research/bert/ $\mathrm{blob} / \mathrm{master} / \mathrm{multilingual.md}$
} 


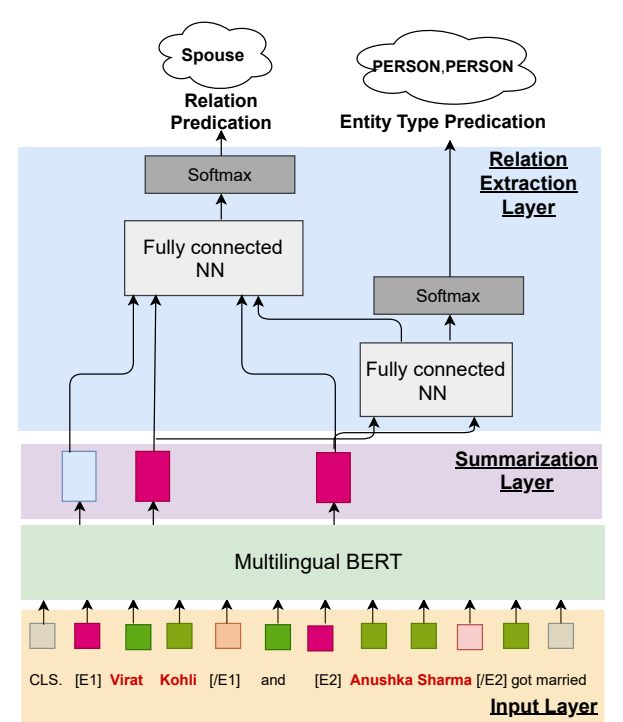

Figure 2: Base RE system, consisting of an input layer, multilingual LM (mBERT), contextual embedding summarization layer, and relation extraction layer.

follows:

$$
\begin{aligned}
& s=\left([\mathrm{CLS}], w_{1}, \ldots,\left[E_{1}\right], e_{1},\left[/ E_{1}\right],\right. \\
& \left.w_{i}, \ldots,\left[E_{2}\right], e_{2},\left[/ E_{2}\right], \ldots,[\mathrm{SEP}]\right)
\end{aligned}
$$

where $e_{1}$ and $e_{2}$ represents mention spans of the two entities of interest and $w_{i}$ represents the $i^{\text {th }}$ token/word. We provide details of annotating the input sequence with special markers for entity types in Appendix C.

\subsection{Summarization layer}

mBERT outputs contextual embeddings $(h)$ for each original token, as well as the special tokens introduced in the input sentence $s$. We pool the contextual information from key positions (Ni et al., 2020) as follows.

Along with CLS which consists of overall sentence information, we also consider the entityspecific information from the entity span tokens and all the tokens between them.

$$
\begin{aligned}
h_{s} & =\left[h_{[\mathrm{CLS}]}, h_{E_{1}}, h_{E_{2}}\right] \\
h_{e_{1}}^{d} & =\underset{i \in\left[E_{1}, \ldots / E_{1}\right]}{\operatorname{avg}} h_{i}^{d}, \quad \forall d \in[1, D] \\
h_{e_{2}}^{d} & =\underset{i \in\left[E_{2}, \ldots / E_{2}\right]}{\operatorname{avg}} h_{i}^{d}, \quad \forall d \in[1, D]
\end{aligned}
$$

where $D$ is the embedding dimension.

We compare two potential summarizing mechanism (a) CLS+ES which consists of infomation in $h_{s}$ and (b) CLS+EN which consists of $h_{[\mathrm{CLS}]}$ and $h_{e_{1}}, h_{e_{2}}$. Other architectural variations are discussed in Appendix C.

\subsection{Relation extraction layer}

Embeddings from the summarization layer can be used in a couple of ways to obtain relation labels.

RE (Relation Extraction): We use CLS + ES or CLS + EN to classify the relations. We do not fine-tune our model for entity classification.

ME (multitasking ensemble): We ensemble the best performance of the following two mechanism per relation.

Without parameter sharing: We use $h_{\mathrm{e}_{1}}$ and $h_{\mathrm{e}_{2}}$ to classify entity types and $h_{s}$ to separately classify relation $r$.

With parameter sharing: We obtain entity type embedding $h_{\mathrm{et}_{1}}$ and $h_{\mathrm{et}_{2}}$ by transforming $h_{\mathrm{e}_{1}}$ and $h_{\mathrm{e}_{2}}$ respectively and use them to predict entity types. We further use them along with $h_{s}$ to predict relation $r$.

\section{Interlingual transfer mechanisms}

By using mBERT, the base RE system already exploits generalized language agnostic information (Pires et al., 2019). In addition, our contextual summaries and type encodings also help map entities and relations in different languages to a common space. Over and above these, we explore transfer through machine translation.

ELFI (Each language for itself): The base RE system is trained using gold instances from the target language, and tested on other instances in the same language. At a high labeling cost, this gives an idea of the maximum achievable RE quality.

LMx (Transfer via mBERT): The base RE system is trained with source language instances and zero target language instances. Then it is tested with target language instances. This is the zeroshot setting LMx0. The whole burden of transfer is on mBERT's language model(LM) in this case. In a related few-shot setting, we allow 1, 5, and 10 sentences per relation from the available gold target instances to fine-tune the RE model. These are called LMx1, LMx5 and LMx10. This lets us calibrate the value of gold target language instances vis-a-vis many training instances in the (resourcerich) source language.

MTx (Model Transfer): Instead of forcing mBERT to do all the heavy lifting, we help out $\mathrm{RE}$ at training time by translating (plentiful) gold source language instances into silver target language instances (as described in Section 2.6) to 
train RE in the target language. This form of translation is widely used for model transfer, see Kozhevnikov and Titov (2014), inter alia. Here, too, we can throw in zero or a few target gold instances, leading to variations we call MTx0, MTx1, MTx5 and MTx10.

Ix (Instance Transfer): Here we go the other way, from the low-resource target to the resourcerich source language, at test time. At training time, we train the base RE system only on English instances (some of which could be translated from target training instances). Each test instance in the target language is translated and aligned (see Appendix B) to English, after which it is processed by the English-trained base RE system. In the zero shot setting Ix0, no gold target instances are used. In a few-shot setting Ix10, we translate 10 gold target sentences per relation to English as additional training data for fine tuning. Our goal is to study the performance gain from Ix0 to Ix10.

\section{Interlingual transfer performance}

For each language, we split the gold data for each relation into training $(80 \%)$ and testing $(20 \%)$ folds. We keep the test fold fixed. We ensure zero overlap in entity pairs for every relation $r$ between the folds, and report on 3-way cross validation. We compare the $\mathbf{R E}$ and $\mathbf{M E}$ task variants and collect macro F1 scores (shown here) and 0/1 accuracy (given in Appendix D). They show the same trend across methods.

\begin{tabular}{l|l|l|l|l}
\hline Summarization & Tasks & Bn & Hi & Te \\
\hline CLS+ES & RE & 92.20 & 94.21 & 72.42 \\
& ME & 92.33 & 94.29 & 72.23 \\
\hline CLS+EN & RE & 92.09 & 93.95 & 73.71 \\
& ME & 91.81 & 94.60 & 75.43 \\
\hline
\end{tabular}

Table 4: RE macro F1 for ELFI.

\subsection{Performance of ELFI}

Table 4 reports macro F1 for ELFI. We observe that both the summarization layer variations yield comparable results. Therefore, for the rest of the experiments, we will report CLS+ES. Multitasking ensembles (ME) provide better accuracy for all the languages. Macro F1 is notably lower for Telugu. After human filtering, several relations fell below 20 instances, resulting in insufficient training.

\begin{tabular}{l|c|r|r|r}
\hline$\downarrow$ Source & Tasks & Bn & Hi & Te \\
\hline ELFI (best) & & 92.33 & 94.60 & 75.43 \\
\hline En & RE & 63.37 & 67.65 & 42.53 \\
& ME & 66.84 & 69.37 & 45.03 \\
\hline \hline Bn & RE & - & 81.45 & 57.01 \\
& ME & - & 80.39 & 58.96 \\
\hline Hi & RE & 76.23 & - & 56.78 \\
& ME & 75.09 & - & 55.14 \\
\hline Te & RE & 60.28 & 60.48 & - \\
& ME & 62.12 & 66.37 & - \\
\hline ALL & RE & 79.68 & 83.45 & 61.58 \\
& ME & 81.06 & 83.54 & 62.00 \\
& & $(-11.27)$ & $(-11.06)$ & $(-13.43)$ \\
\hline
\end{tabular}

Table 5: Macro F1 of LMx0. Green represents best and Red represents the worst performance compared to ELFI. Yellow represents the best performance for a single source language.

\subsection{Performance of LMx0}

Table 5 shows LM-based transfer results. Of considerable interest is the pairing of (source, target) languages that result in best performance.

- Source Hi helps to achieve the best macro F1 score for target $\mathrm{Bn}$, compared to other languages. Conversely, $\mathrm{Bn}$ is the best source for Hi. This may be explained by their membership in the Indo-European language genus.

- In contrast, Te, belonging to the Dravidian genus, is indifferent to Hi vs. Bn.

- Source En performs poorly for all three Indian languages. So it seems better for Indian languages to assist each other for RE, compared to importing foreign help.

- That being said, the best target performance is uniformly achieved by training on all sources except the target, which contributes diversity from multiple language families and builds a robust RE model.

- However, this best performance is still substantially behind ELFI performance (last row) for all languages.

\subsection{Performance of $\operatorname{LMx}\{1,5,10\}$}

Table 6 shows the effect of allowing a small percentage of available gold instances in the target language for training. Understandably, revealing more instances is better; LMx10 gives the best target performance. The deficit from ELFI reduces steadily with more 'shots'. Otherwise the trends from $\mathrm{LMx} 0$ prevail. $\mathrm{Bn}$ and $\mathrm{Hi}$ are best partners, and both of them show similar performance as a source for $\mathrm{Te}$. Incorporating all-but-target (ALL) languages gives further performance boost. The improvements show that TransRel can learn target-specific features with very few examples. 


\begin{tabular}{l|c|c|c|c|c|c|c|c|c|c}
\hline$\downarrow$ Source & Tasks & \multicolumn{3}{|c|}{ Bn } & \multicolumn{3}{c}{ Hi } & \multicolumn{3}{c}{ Te } \\
\hline & & LMx1 & LMx5 & LMx10 & LMx1 & LMx5 & LMx10 & LMx1 & LMx5 & LMx10 \\
\hline En & RE & 68.89 & 78.37 & 82.30 & 72.25 & 78.95 & 82.48 & 47.12 & 57.11 & 57.40 \\
& ME & 73.30 & 81.97 & 84.03 & 75.72 & 83.82 & 84.72 & 51.04 & 58.91 & 63.20 \\
\hline Bn & RE & - & - & - & 79.80 & 85.02 & 89.83 & 61.18 & 64.10 & 70.14 \\
& ME & - & - & - & 80.82 & 87.71 & 90.74 & 62.67 & 67.34 & 69.80 \\
\hline Hi & RE & 77.64 & 83.38 & 84.55 & - & - & - & 64.24 & 63.98 & 68.21 \\
& ME & 79.73 & 84.51 & 84.02 & - & - & - & 64.02 & 68.51 & 71.72 \\
\hline Te & RE & 71.78 & 78.48 & 83.73 & 73.34 & 78.22 & 83.76 & - & - & - \\
& ME & 73.54 & 79.52 & 82.23 & 71.57 & 79.83 & 85.21 & - & - & - \\
\hline ALL & RE & 81.09 & 85.22 & 86.18 & 86.07 & 88.35 & 90.55 & 61.95 & 67.87 & 68.18 \\
& ME & 83.77 & 87.71 & 88.57 & 86.36 & 89.99 & 91.80 & 66.68 & 69.99 & 71.23 \\
& $(-8.56)$ & $(-4.52)$ & $(-3.76)$ & $(-8.24)$ & $(-4.61)$ & $(-2.8)$ & $(-8.55)$ & $(-5.44)$ & $(-4.23)$ \\
\hline \hline LMx0 (best) & \multicolumn{9}{|c|}{81.06} \\
\hline
\end{tabular}

Table 6: Macro F1 of $\operatorname{LMx}\{1,5,10\}$ for different source and target languages. Green denotes performance gain (darker denotes larger gain) with respect to LMx0 (last row) for a given target language. Gap from ELFI for the best performing cell is in parentheses.

\begin{tabular}{|c|c|c|c|c|c|}
\hline$\downarrow$ Target & Tasks & MTx0 & MTx1 & MTx5 & MTx10 \\
\hline \multirow[t]{2}{*}{ Bn } & $\mathrm{RE}$ & 66.22 & 66.29 & 79.10 & 82.38 \\
\hline & $\mathrm{ME}$ & 69.99 & 71.62 & 80.03 & $\begin{array}{l}85.06 \\
(-7.27)\end{array}$ \\
\hline \multirow[t]{2}{*}{$\mathbf{H i}$} & $\mathrm{RE}$ & 73.35 & 77.62 & 84.05 & 86.46 \\
\hline & ME & 74.84 & 81.75 & 85.95 & 87.14 \\
\hline \multirow[t]{2}{*}{ Te } & $\mathrm{RE}$ & 51.89 & 51.13 & 58.87 & 61.34 \\
\hline & ME & 50.25 & 58.81 & 60.75 & $\begin{array}{l}66.37 \\
(-9.06)\end{array}$ \\
\hline
\end{tabular}

Table 7: Macro F1 for MTx $\{0,1,5,10\}$. Performance gap between best performance (Green) and ELFI is shown in parentheses.

\subsection{MTx performance}

Here we investigate the importance of silver instances in a low-resource scenario. In the absence of abundant target instances for ELFI-style training, we can leverage potentially plentiful instances from a source language, namely, En. Table 7 shows the results.

- Even after using all available En gold instances to generate silver target instances, adding gold target instances gives steady improvements.

- At 10 instance per relation gold exposure, there is still a gap of $7.27 \%$ for $\mathrm{Bn}, 7.46 \%$ for $\mathrm{Hi}$ and $9.06 \%$ for Te compared to ELFI.

This gives a strong idea of the worth of gold target instances, and motivates importing from the optimal languages - an optimization that opens up a significant avenue of future work.

\section{Performance of $\mathrm{Ix}$}

Here we compare the performance of instance transfer Ix with that of the model transfer LMx. We experiment using the zero-shot and 10-shot settings with RE and ME. In Table 8, for each setting, we report 10 -shot performance with the gain obtained from its zero-shot counterpart in parentheses. Ix0 performs better than LMx0 for all sourcetarget settings. High quality translations of well formed instances play a crucial role in the performance gain of instance transfer. However, the gain of 10-shot transfer over zero-shot in Ix is less than that of LMx. One possible reason could be that in the Ix setting, because we translate everything to English, few-shot examples hardly add any language specific information; while in the case of LMx, few-shot examples are likely to add more target language specific information to the model.

\section{Augmented FewRel comparison}

Here we investigate the effectiveness of extending an existing RE dataset for Indian languages. Existing supervised dataset FewRel (Han et al., 2018) has 27 common relations with IndoRE.

\begin{tabular}{l|c|l|l|l}
\hline$\downarrow$ Target & \multicolumn{2}{|c|}{ FewRel } & \multicolumn{1}{c}{ IndoRE (ours) } \\
\hline & $\begin{array}{l}\text { Training } \\
\text { instances }\end{array}$ & $\begin{array}{l}\text { Macro } \\
\text { F1 }\end{array}$ & $\begin{array}{l}\text { Training } \\
\text { instances }\end{array}$ & $\begin{array}{l}\text { Macro } \\
\text { F1 }\end{array}$ \\
\hline & \multicolumn{4}{|c}{ ELFI } \\
\hline Bn & 18277 & 84.19 & 3845 & 92.46 \\
\hline $\mathbf{H i}$ & 18387 & 91.32 & 2987 & 96.45 \\
\hline $\mathbf{T e}$ & 18462 & 74.66 & 5136 & 79.45 \\
\hline \hline Bn & 18900 & 80.26 & 3692 & 89.27 \\
\hline $\mathbf{H i}$ & 18900 & 90.78 & 2944 & 96.41 \\
\hline $\mathbf{T e}$ & 18900 & 69.57 & 5101 & 80.38 \\
\hline
\end{tabular}

Table 9: Macro F1 training with FewRel vs. IndoRE.

We translate the En evidence sentences released by them to Bn, Hi and Te using Google Translate API, and map the entities in the translated sentences. In Table 9 we report macro F1 obtained on the 27 relations while our model (CLS+ES) is trained with FewRel translated data, vs. our gold data in ELFI setting. We use $80 \%$ of our data to train and $20 \%$ to test. We use the whole FewRel data for 27 relations for training and use the same test split of ours to evaluate the performance. We observe that there is a performance gain greater 


\begin{tabular}{|c|c|c|c|c|c|c|c|}
\hline$\downarrow$ Source & Task & & & & & & \\
\hline & & LMx10 & Ix10 & LMx10 & Ix10 & LMx10 & Ix10 \\
\hline & RE & 82.30 & 84.14 & 82.48 & 86.30 & 57.40 & 70.18 \\
\hline En & & $(+18.93)$ & $(+9.60)$ & $(+14.83)$ & $(+7.39)$ & $(+14.87)$ & $(+9.36)$ \\
\hline & ME & $\begin{array}{l}84.03 \\
(+1719)\end{array}$ & $\begin{array}{l}84.29 \\
(+906)\end{array}$ & $\begin{array}{l}84.72 \\
(+1535)\end{array}$ & $\begin{array}{l}87.05 \\
(+610)\end{array}$ & $\begin{array}{l}63.20 \\
(+1817)\end{array}$ & $\begin{array}{l}71.01 \\
(+073)\end{array}$ \\
\hline & & & & 89.83 & 89.98 & 70.14 & 75.83 \\
\hline Bn & RE & - & - & $(+8.38)$ & $(+4.04)$ & $(+13.04)$ & $(+2.70)$ \\
\hline & & - & $\begin{array}{lll}- & -\end{array}$ & 90.74 & 89.74 & 69.80 & 79.10 \\
\hline & & 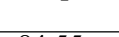 & 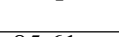 & $(+10.35)$ & $(+3.32)$ & $(+8.84)$ & $(+4.44)$ \\
\hline $\mathrm{Hi}$ & $\mathrm{RE}$ & $\begin{array}{l}84.55 \\
(+832)\end{array}$ & $\begin{array}{l}85.61 \\
(+4.78)\end{array}$ & - & - & $\begin{array}{l}68.21 \\
(+1143)\end{array}$ & $\begin{array}{l}75.36 \\
(+0.26)\end{array}$ \\
\hline & & 84.02 & 85.95 & & & 71.72 & 78.73 \\
\hline & ME & $(+8.93)$ & $(+4.65)$ & 0 & & $(+16.58)$ & $(+2.83)$ \\
\hline Te & $\mathrm{RE}$ & $\begin{array}{l}83.73 \\
(+2345)\end{array}$ & $\begin{array}{l}81.94 \\
(+632)\end{array}$ & $\begin{array}{l}83.76 \\
(+2328)\end{array}$ & $\begin{array}{l}86.54 \\
(+783)\end{array}$ & - & - \\
\hline 10 & & $\begin{array}{l}(+23.45) \\
82.23\end{array}$ & $\begin{array}{l}(+0.32) \\
83.53\end{array}$ & $\begin{array}{l}(+23.28) \\
85.21\end{array}$ & $\begin{array}{l}+1.83) \\
87.19\end{array}$ & & \\
\hline & ME & $(+20.11)$ & $(+10.98)$ & $(+18.84)$ & $(+6.89)$ & $\begin{array}{ll}- \\
\end{array}$ & \\
\hline AII & $\mathrm{RE}$ & $\begin{array}{l}86.18 \\
(+650\end{array}$ & $\begin{array}{l}88.07 \\
(+275)\end{array}$ & $\begin{array}{l}90.55 \\
(+710)\end{array}$ & $\begin{array}{l}90.93 \\
(-0.62)\end{array}$ & $\begin{array}{l}68.18 \\
(+660)\end{array}$ & $\begin{array}{l}78.74 \\
(+210)\end{array}$ \\
\hline & & 88.57 & 89.08 & 91.80 & 91.66 & 71.23 & 80.37 \\
\hline & MF & $(+7.51)$ & $(+1.98)$ & $(+8.26)$ & $(+1.02)$ & $(+9.23)$ & $(+1.26)$ \\
\hline
\end{tabular}

Table 8: Macro F1 comparison between LMx10 and Ix10 for different source and target languages. The corresponding gains from zeroshot is reported in parentheses.

than $5 \%$ in all the three languages, with highest gain achieved in Te, while using far fewer labeled instances. This underscores the necessity of developing RE datasets. In the instance transfer setup we translate the common relation instances of IndoRE to English and report the results in the last three rows of Table 9 . We observe more than $6 \%$ gain in all languages.

\section{Transfer error analysis}

We investigate different type of errors occurring in LMx and Ix setting. Table 10 shows some of the error types with examples. In case of LMx, we sometimes find that the prediction is sensitive to changes in one or two words, while the Ix counterpart is more robust, as we can see in the first example in Table 10. The second example shows that the words between the two entities also play a significant role in prediction; e.g., if a word strongly associated with the relation is present in between the two entities, the prediction is more accurate compared to when the word is at some other part of the sentence. Unavailability of diverse data while training the LMx model may be the main contributing factor for such errors. We build our RE model using mBERT, which has larger exposure to English compared to low-resource Indian languages. This possibly helps Ix perform better than LMx. Word reordering may help Google Translate to be more accurate, which in turn boosts Ix performance, as we see in the third example in Table 10. As we can see in the last example the major reason behind the occasional poor prediction of Ix is the incomplete or wrong translation with erroneous en- tity mapping from the target sentence.

\section{Related work on RE systems}

Named entity recognition and relation extraction have been of continued interest in recent years. In this section we discuss relevant work that propose different architectures for increasingly accurate named entity and relation prediction. Zheng et al. (2017) jointly extract entities and relations based on a novel tagging scheme. Chen et al. (2020) propose joint named entity recognition and relation extraction training in the clinical analytic space. In a similar direction, Wang and $\mathrm{Lu}$ (2020) propose table-sequence encoders for joint training. Bekoulis et al. (2018) use adversarial training for joint entity and relation extraction. Recently, a BERT-based model (Eberts and Ulges, 2019) used span-based encoding for joint extraction. All of these approaches show promising improvements in $\mathrm{RE}$ from joint $\mathrm{NER}+\mathrm{RE}$ training in comparison to standalone RE for monolingual data. Fu et al. (2019) build a dependency graph and use a GCN to infer necessary information. The BiLSTM-CRFbased entity recognition model (Nguyen and Verspoor, 2019) with a deep biaffine attention layer to model second-order interactions among latent features for relation classification shows great performance for English relation extraction. However, these models incorporate specific building blocks (e.g., the dependency graph) which are not easy to procure with high accuracy in case of low-resource languages. 


\begin{tabular}{|c|c|c|}
\hline Observation & Test Instance (Prediction - LMx) & Test Instance (Prediction - Ix) \\
\hline \multirow[t]{2}{*}{$\begin{array}{l}\text { Ground Truth: } \\
\text { composer }\end{array}$} & $\begin{array}{l}\text { आनंद समाराकून, (1911-1962), श्रीलंका के एक संगीतकार, } \\
\text { कवि और शिक्षक थे समाराकून ने श्रीलंका के राष्ट्रगान श्रीलंका माता } \\
\text { की रचना की थी और उन्हें कलात्मक श्रीलंकाई संगीत का पिता माना जाता है } \\
\text { Prediction - screenwriter }\end{array}$ & $\begin{array}{l}\text { Ananda Samarakoon, (1911-1962), was a Sri Lankan musician, } \\
\text { poet and teacher. Samarakoon composed the Sri Lankan } \\
\text { national anthem Sri Lanka Mata and is considered the father } \\
\text { of artistic Sri Lankan music. } \\
\text { Prediction - composer }\end{array}$ \\
\hline & $\begin{array}{l}\text { आनंद समाराकून, (1911-1962), श्रीलंका के एक संगीतकार, } \\
\text { कवि और शिक्षक थे समाराकून ने श्रीलंका के राष्ट्रगान श्रीलंका पिता } \\
\text { की रचना की थी और उन्हें कलात्मक श्रीलंकाई संगीत का पिता माना जाता है } \\
\text { Prediction - child }\end{array}$ & $\begin{array}{l}\text { Ananda Samarakoon,(1911-1962), was a Sri Lankan musician, } \\
\text { poet and teacher. Samarakoon composed the Sri Lankan } \\
\text { national anthem Sri Lanka Father and is considered the father } \\
\text { of artistic Sri Lankan music. } \\
\text { Prediction composer }\end{array}$ \\
\hline \multirow[t]{2}{*}{$\begin{array}{l}\text { LMx prediction } \\
\text { depends on } \\
\text { word ordering } \\
\text { Ground Truth: } \\
\text { child }\end{array}$} & $\begin{array}{l}\text { হিন্দুদের ধর্মগ্রন্থ ও মহাকাব্য মহাভারত অনুযায়ী ভীপ্মক } \\
\text { ছিলেন বিদর্ভের রাজা এবং কৃষ্ণের প্রথমা পত্নী রুক্মিণীর পিতা। } \\
\text { Prediction - mother }\end{array}$ & $\begin{array}{l}\text { According to Hindu scriptures and the epic Mahabharata, } \\
\text { Bhishmak was the king of Vidarbha and the father of } \\
\text { Krishna's first wife Rukmini. } \\
\text { Prediction - child }\end{array}$ \\
\hline & $\begin{array}{l}\text { হিন্দুদের ধর্মগ্রন্থ ও মহাকাব্য মহাভারত অনুযায়ী বিদর্ভের রাজা এবং } \\
\text { কৃষ্ণের প্রথমা পত্নী রুক্মিণীর পিতা ছিলেন ভীপ্মক } \\
\text { Prediction - child }\end{array}$ & $\begin{array}{l}\text { According to the Hindu scriptures and the epic Mahabharata, } \\
\text { Bhishmak was the father of Rukmini the king of Vidarbha and } \\
\text { Krishna's first wife. } \\
\text { Prediction - child }\end{array}$ \\
\hline \multirow[t]{2}{*}{$\begin{array}{l}\text { Change in word } \\
\text { ordering helps } \\
\text { both LMx and Ix } \\
\text { Ground Truth: } \\
\text { child }\end{array}$} & $\begin{array}{l}\text { বলিউডে এখনও নহুন মুখ হিসাবেই বিবেচিত হন জাহ্ববী কাপুর } \\
\text { মাত্র দুটি ছবি এবং একটি শর্টফিল্মে অভিনয় করেছেন শ্রীদেবী কন্যা } \\
\text { Prediction - spouse }\end{array}$ & $\begin{array}{l}\text { Jahnavi Kapoor is still considered a new face in Bollywood. } \\
\text { Sridevi Kanya has acted in only two films and one short film. } \\
\text { Prediction - performer }\end{array}$ \\
\hline & $\begin{array}{l}\text { বলিউডে এখনও নহুন মুখ হিসাবেই বিবেচিত হন শ্রীদেবী কন্যা } \\
\text { জাহ্বীকাপুর মাত্র দুটি ছবি এবং একটি শর্টিফিল্মে অভিনয় করেছেন } \\
\text { Prediction - child }\end{array}$ & $\begin{array}{l}\text { Sridevi's daughter Jahnavi Kapoor, who is still considered a } \\
\text { new face in Bollywood, has acted in only two films and one } \\
\text { short film. } \\
\text { Prediction - child }\end{array}$ \\
\hline $\begin{array}{l}\text { Ix prediction } \\
\text { depends on } \\
\text { translation quality } \\
\text { Ground Truth: } \\
\text { award_received }\end{array}$ & $\begin{array}{l}\text { কানাইলাল শীল (৫ সেপ্টেম্বর, ১b৯৫-২০ জুলাই, ১৯৭৪) ছিলেন } \\
\text { একজন বাঙালি দোতারাবাদক, সুরকার, লোকসঙ্গীত রচয়িতা ও } \\
\text { সংগ্রাহক, লোকসঙ্গীতে অবদানের জন্য বাংলাদেশ সরকার তাকে } \\
\text { ১৯৮৭ সালে মরণোত্তর একুশে পদক ভূষিত করে } \\
\text { Prediction - award_received }\end{array}$ & $\begin{array}{l}\text { Kanailal Sheel (September 5, } 1895 \text { - July 20,1984) was a } \\
\text { Bengali dotara player, composer, composer and collector } \\
\text { of folk music. } \\
\text { Prediction - occupation } \\
\text { Correct translation - Kanailal Sheel (September 5, 1895- } \\
\text { July 20,1974) was a Bengali dotaraplayer, composer, folk } \\
\text { music composer and collector, who was posthumously } \\
\text { awarded the Ekushey Medal by the Government of Bangladesh } \\
\text { in } 1987 \text { for his contribution to folk music. }\end{array}$ \\
\hline
\end{tabular}

Table 10: Error analysis of transfer mechanisms.

\section{Conclusion}

In this work, we release IndoRE, a rich set of goldtagged sentences for NER and relation extraction in En, Bn, Hi and Te. We also present TransRel, a multilingual joint NER and RE system with interlingual transfer. Our experiments show promising results on generalization and knowledge transfer. Even in the absence of gold training data for a given language, instances from other languages can contribute to considerable RE accuracy boosts. We uncover intuitive patterns in the extent of improvements conferred by one language on another. We believe IndoRE and TransRel can significantly assist future study of Indian language relation extraction.

\section{References}

Giannis Bekoulis, Johannes Deleu, Thomas Demeester, and Chris Develder. 2018. Adversarial training for multi-context joint entity and relation extraction. In Proceedings of the 2018 Conference on Empirical Methods in Natural Language Processing, pages 2830-2836, Brussels, Belgium. Association for Computational Linguistics.
Miao Chen, Ganhui Lan, Fang Du, and Victor Lobanov. 2020. Joint learning with pre-trained transformer on named entity recognition and relation extraction tasks for clinical analytics. In Proceedings of the 3rd Clinical Natural Language Processing Workshop, pages 234-242, Online. Association for Computational Linguistics.

Alexis Conneau et al. 2020. Unsupervised crosslingual representation learning at scale.

Markus Eberts and Adrian Ulges. 2019. Span-based joint entity and relation extraction with transformer pre-training.

Tsu-Jui Fu, Peng-Hsuan Li, and Wei-Yun Ma. 2019. GraphRel: Modeling text as relational graphs for joint entity and relation extraction. In Proceedings of the 57th Annual Meeting of the Association for Computational Linguistics, pages 1409-1418, Florence, Italy. Association for Computational Linguistics.

Xu Han, Hao Zhu, Pengfei Yu, Ziyun Wang, Yuan Yao, Zhiyuan Liu, and Maosong Sun. 2018. FewRel: A large-scale supervised few-shot relation classification dataset with state-of-the-art evaluation. In Proceedings of the 2018 Conference on Empirical Methods in Natural Language Processing, pages 48034809, Brussels, Belgium. Association for Computational Linguistics.

Iris Hendrickx, Su Nam Kim, Zornitsa Kozareva, Preslav Nakov, Diarmuid Ó Séaghdha, Sebastian 
Padó, Marco Pennacchiotti, Lorenza Romano, and Stan Szpakowicz. 2010. SemEval-2010 task 8: Multi-way classification of semantic relations between pairs of nominals. In Proceedings of the 5th International Workshop on Semantic Evaluation, pages 33-38, Uppsala, Sweden. Association for Computational Linguistics.

Divyanshu Kakwani, Anoop Kunchukuttan, Satish Golla, Gokul N.C., Avik Bhattacharyya, Mitesh M. Khapra, and Pratyush Kumar. 2020. IndicNLPSuite: Monolingual corpora, evaluation benchmarks and pre-trained multilingual language models for Indian languages. In Findings of the Association for Computational Linguistics: EMNLP 2020, pages 49484961, Online. Association for Computational Linguistics.

Mikhail Kozhevnikov and Ivan Titov. 2014. Crosslingual model transfer using feature representation projection. In Proceedings of the 52nd Annual Meeting of the Association for Computational Linguistics (Volume 2: Short Papers), pages 579-585, Baltimore, Maryland. Association for Computational Linguistics.

Mike Mintz, Steven Bills, Rion Snow, and Daniel Jurafsky. 2009. Distant supervision for relation extraction without labeled data. In Proceedings of the Joint Conference of the 47th Annual Meeting of the ACL and the 4th International Joint Conference on Natural Language Processing of the AFNLP, pages 10031011, Suntec, Singapore. Association for Computational Linguistics.

Dat Quoc Nguyen and Karin Verspoor. 2019. End-toend neural relation extraction using deep biaffine attention. Advances in Information Retrieval, page 729-738.

Jian Ni, Taesun Moon, Parul Awasthy, and Radu Florian. 2020. Cross-lingual relation extraction with transformers.

Telmo Pires, Eva Schlinger, and Dan Garrette. 2019. How multilingual is multilingual bert?

Sebastian Riedel, Limin Yao, and Andrew McCallum. 2010. Modeling relations and their mentions without labeled text. In Machine Learning and Knowledge Discovery in Databases, pages 148-163.

Livio Baldini Soares, Nicholas FitzGerald, Jeffrey Ling, and Tom Kwiatkowski. 2019. Matching the blanks: Distributional similarity for relation learning.

Jue Wang and Wei Lu. 2020. Two are better than one: Joint entity and relation extraction with tablesequence encoders. In Proceedings of the 2020 Conference on Empirical Methods in Natural Language Processing (EMNLP), pages 1706-1721, Online. Association for Computational Linguistics.
Yuhao Zhang, Victor Zhong, Danqi Chen, Gabor Angeli, and Christopher D. Manning. 2017. Positionaware attention and supervised data improve slot filling. In Proceedings of the 2017 Conference on Empirical Methods in Natural Language Processing, pages 35-45, Copenhagen, Denmark. Association for Computational Linguistics.

Suncong Zheng, Feng Wang, Hongyun Bao, Yuexing Hao, Peng Zhou, and Bo Xu. 2017. Joint extraction of entities and relations based on a novel tagging scheme. In Proceedings of the 55th Annual Meeting of the Association for Computational Linguistics (Volume 1: Long Papers), pages 1227-1236, Vancouver, Canada. Association for Computational Linguistics. 


\section{A Data Bootstrapping Recipe for Low-Resource Multilingual Relation Classification (Appendix)}

\section{A Complexity of relations}

Figure 3 shows the average lexical distance between entities accross relations as a measure of complexity.

\section{B Annotating translated sentences}

Starting from gold annotated sentences in the source language, it is nontrivial to transfer or 'project' the gold entity spans to the sentence after translation to the target language. The steps we follow to do this are shown in Algorithm 1.

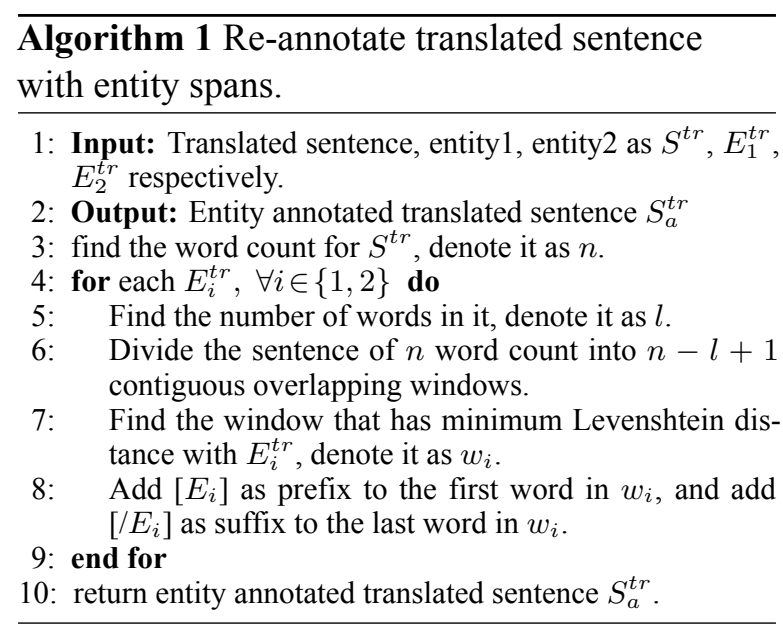

\section{Base model variations}

The best performing configuration of the base RE system is described in Figure 2. In this section, we briefly describe the other variations we evaluated.

\section{C.1 Input layer}

We append special markers to the input sentence $(s)$ for useful embedding generation by mLMs in different ways other than the best performing one reported in main paper.

Entity type markers (ET): Mark entity spans by special tokens denoting their type as follows:

$$
\begin{aligned}
& s=\left([\mathrm{CLS}], w_{1}, \ldots,\left[E T_{1}\right], \text { entity },\left[/ E T_{1}\right],\right. \\
& \left.w_{i}, \ldots,\left[E T_{2}\right], \text { entity },\left[/ E T_{2}\right], \ldots,[\mathrm{SEP}]\right)
\end{aligned}
$$

Here $E T_{1}, E T_{2}$ are the entity types of the corresponding entities.

Entity span and type markers (EST): We mark both the entity number ( 1 or 2$)$ and their types:

$$
\begin{aligned}
& s=\left([\mathrm{CLS}], w_{1}, \ldots,\left[E_{1}\right],\left[E T_{1}\right], \text { entity },\right. \\
& {\left[/ E T_{1}\right],\left[/ E_{1}\right], w_{i}, \ldots,\left[E_{2}\right],\left[E T_{2}\right],} \\
& \text { entity } \left.,\left[/ E T_{2}\right],\left[/ E_{2}\right], \ldots,[\mathrm{SEP}]\right)
\end{aligned}
$$

Language identity marker (L): We append the language identifier token $[L]$, where $L$ is in $\{\mathrm{En}, \mathrm{Bn}, \mathrm{Hi}, \mathrm{Te}\})$ after the [CLS] token for all of the above cases:

$$
\begin{aligned}
& s=\left([\mathrm{CLS}],[L], w_{1}, \ldots,\left[E_{1}\right],\right. \text { entity, } \\
& {\left[/ E_{1}\right], w_{i}, \ldots,\left[E_{2}\right], \text { entity },\left[/ E_{2}\right],} \\
& \ldots,[\mathrm{SEP}])
\end{aligned}
$$

\section{C.2 Embedding layer}

We experimented with three different multilingual language models (mLMs), namely, mBERT $^{9}$, XLM-R (Conneau et al., 2020) and IndicBERT (Kakwani et al., 2020) to leverage the pretrained multilingual embedding space. mBERT was generally the best.

\section{C.3 Summarization layer}

The mLM generates embeddings $(h)$ for each word as well as special tokens introduced in different input formats given a sentence $s$. As these mLMs provide contextual embeddings, they are expected to emit useful and different information corresponding to different special tokens. Here we describe several information pooling mechanisms to evaluate the effectiveness of special tokens, other than the ones described in the main paper.

CLS: Here we consider CLS token embedding which encodes necessary contextual information about the sentence.

$$
h_{s}=h_{[\mathrm{CLS}]}
$$

ES: Here we only consider the entity specific information from special entity span tokens $\left[E_{1} \ldots / E_{1}\right]$ and $\left[E_{2} \ldots / E_{2}\right]$.

$$
h_{s}=\left[h_{E_{1}}, h_{E_{2}}\right]
$$

\section{Other performance measures}

In the main paper we reported macro averaged $\mathrm{F} 1$ across labels. Here we also provide micro averaged $0 / 1$ accuracy metric. The broad trends remain

\footnotetext{
${ }^{9}$ https://github.com/google-research/bert/ $\mathrm{blob} / \mathrm{master} / \mathrm{multilingual.md}$
} 


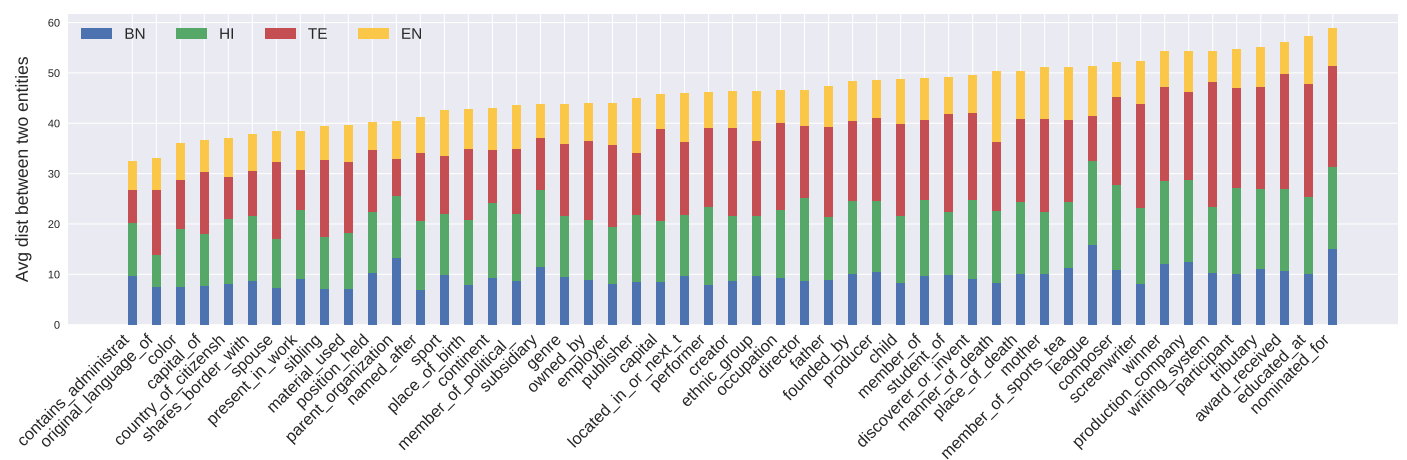

Figure 3: Average lexical distance between entities across relation and languages. All relations in En and Bn have small lexical distance. Most relations need large lexical distances for Te and Hi.

the same. In the Table 11 we report the accuracy for ELFI setting in three languages. In the Table 12 we report the accuracy obtain in different sourcetarget language pair in zero-shot setting. Table 15 shows the accuracy for LMx few-shot transfer. Table 16 shows the accuracy comparison between LMx0,10 with Ix0,10. Table 13 shows zero-shot, few-shot accuracy for different target languages in MTx setting. And Table 14 shows the accuracy comparison between FewRel and IndoRE for both ELFI and Ix.

\begin{tabular}{l|l|l|l|l}
\hline $\begin{array}{l}\text { Summerization } \\
\text { Layer }\end{array}$ & Model & BN & HI & TE \\
\hline CLS+ES & RE & 93.01 & 95.13 & 85.91 \\
& ME & 93.75 & 95.13 & 87.12 \\
\hline CLS+EN & RE & 92.83 & 94.69 & 85.91 \\
& ME & 93.21 & 95.42 & 86.94 \\
\hline
\end{tabular}

Table 11: Microaveraged 0/1 accuracy of ELFI.

\begin{tabular}{l|l|l|l|l}
\hline Train & Model & BN & HI & TE \\
\hline self & & 93.75 & 95.42 & 87.12 \\
\hline EN & RE & 65.75 & 71.82 & 52.02 \\
& ME & 70.96 & 75.38 & 55.87 \\
\hline BN & RE & - & 85.40 & 67.85 \\
& ME & - & 84.10 & 68.09 \\
\hline HI & RE & 77.70 & - & 61.59 \\
& ME & 78.00 & - & 62.01 \\
\hline TE & RE & 64.64 & 67.03 & - \\
& ME & 66.48 & 72.69 & - \\
\hline ALL & RE & 81.19 & 87.22 & 68.33 \\
& ME & 82.48 & 86.93 & 70.62 \\
\hline
\end{tabular}

Table 12: Performance (microaveraged accuracy) of zero-shot training on different languages. Green represents closest performance and Red represents the most deviating performance compared to self training. Yellow represents the best performance among single language input.

\begin{tabular}{l|c|c|c|c|c}
\hline$\downarrow$ Target & Task & 0-shot & 1 shot & 5 shot & 10 shot \\
\hline BN & RE & 70.04 & 69.18 & 80.70 & 84.01 \\
& ME & 72.79 & 74.75 & 81.00 & $86.34(-7.41)$ \\
\hline HI & RE & 76.32 & 80.75 & 85.62 & 87.51 \\
& ME & 78.50 & 84.46 & 88.02 & $88.67(-6.75)$ \\
\hline TE & RE & 60.26 & 60.63 & 69.96 & 69.90 \\
& ME & 57.62 & 66.83 & 69.90 & $73.45(-13.67)$ \\
\hline
\end{tabular}

Table 13: Performance (microaveraged accuracy) for silver training (MTx) for different target languages. The gaps between the best performance and ELFI are shown in parentheses.

\begin{tabular}{l|c|c|c|c}
\hline$\downarrow$ Target & \multicolumn{2}{|c|}{ FewRel } & \multicolumn{2}{c}{ IndoRE (ours) } \\
\hline & $\begin{array}{l}\text { Training } \\
\text { instances }\end{array}$ & Accuracy & $\begin{array}{l}\text { Training } \\
\text { instances }\end{array}$ & Accuracy \\
\hline \multicolumn{4}{|c}{ ELFI } \\
\hline $\mathbf{B n}$ & 18277 & 85.20 & 3845 & 92.39 \\
\hline $\mathbf{H i}$ & 18387 & 91.82 & 2987 & 96.46 \\
\hline $\mathbf{T e}$ & 18462 & 76.81 & 5136 & 85.55 \\
\hline \hline $\mathbf{5 n}$ & 18900 & 82.93 & 3692 & 89.93 \\
\hline $\mathbf{H i}$ & 18900 & 91.86 & 2944 & 96.55 \\
\hline $\mathbf{T e}$ & 18900 & 74.60 & 5101 & 85.71 \\
\hline
\end{tabular}

Table 14: Accuracy training with FewRel vs. IndoRE. 


\begin{tabular}{l|c|c|c|c|c|c|c|c|c|c}
\hline$\downarrow$ Source & Tasks & \multicolumn{3}{|c|}{ Bn } & \multicolumn{3}{c|}{ Hi } & \multicolumn{3}{c}{ Te } \\
\hline \multirow{2}{*}{ En } & & LMx1 & LMx5 & LMx10 & LMx1 & LMx5 & LMx10 & LMx1 & LMx5 & LMx10 \\
& RE & 71.57 & 80.02 & 83.95 & 76.69 & 81.84 & 84.46 & 55.57 & 61.29 & 64.90 \\
& ME & 75.12 & 83.88 & 84.99 & 80.10 & 85.19 & 86.20 & 61.53 & 65.86 & 70.74 \\
\hline Bn & RE & - & - & - & 83.88 & 86.71 & 90.85 & 67.43 & 69.48 & 75.68 \\
& ME & - & - & - & 84.82 & 88.67 & 91.79 & 69.72 & 70.80 & 76.94 \\
\hline Hi & RE & 79.66 & 85.23 & 85.97 & - & - & - & 68.93 & 69.66 & 72.43 \\
& ME & 82.05 & 85.42 & 85.48 & - & - & - & 68.99 & 71.88 & 75.14 \\
\hline Te & RE & 73.65 & 80.15 & 85.11 & 78.29 & 81.70 & 86.27 & - & - & - \\
& ME & 74.82 & 80.76 & 83.64 & 77.05 & 82.43 & 87.36 & - & - & - \\
\hline ALL & RE & 82.10 & 86.64 & 87.68 & 88.23 & 89.54 & 91.50 & 70.38 & 73.15 & 76.10 \\
& ME & 84.74 & 88.42 & 89.83 & 87.73 & 91.19 & 92.74 & 72.07 & 74.17 & 78.75 \\
& & $(-9.01)$ & $(-5.33)$ & $(-3.92)$ & $(-7.69)$ & $(-4.23)$ & $(-2.68)$ & $(-15.05)$ & $(-12.95)$ & $(-8.37)$ \\
\hline \hline
\end{tabular}

Table 15: Accuracy of $\operatorname{LMx}\{1,5,10\}$ for different source and target languages. Green denotes performance gain (darker denotes larger gain) with respect to LMx0 (last row) for a given target language. Gap from ELFI for the best performing cell is in parentheses.

\begin{tabular}{|c|c|c|c|c|c|c|c|}
\hline \multirow[t]{2}{*}{$\downarrow$ Source } & \multirow[t]{2}{*}{ Task } & \multicolumn{2}{|c|}{ Bn } & \multicolumn{2}{|c|}{ Hi } & \multicolumn{2}{|c|}{ Te } \\
\hline & & LMx10 & Ix 10 & LMx10 & Ix 10 & LMx10 & Ix 10 \\
\hline \multirow{2}{*}{ En } & $\mathrm{RE}$ & $\begin{array}{l}83.95 \\
(+1825)\end{array}$ & $\begin{array}{l}85.43 \\
(+899)\end{array}$ & $\begin{array}{l}84.46 \\
(+12.64)\end{array}$ & $\begin{array}{l}88.69 \\
(+628)\end{array}$ & $\begin{array}{l}64.90 \\
(+1288)\end{array}$ & $\begin{array}{l}77.08 \\
(+922)\end{array}$ \\
\hline & $\mathrm{ME}$ & $\begin{array}{l}84.99 \\
(+14.03)\end{array}$ & $\begin{array}{l}85.50 \\
(+8.62)\end{array}$ & $\begin{array}{l}86.20 \\
(+10.82)\end{array}$ & $\begin{array}{l}88.69 \\
(+5.76)\end{array}$ & $\begin{array}{l}70.74 \\
(+14.87)\end{array}$ & $\begin{array}{l}77.47 \\
(+8.13)\end{array}$ \\
\hline \multirow[t]{2}{*}{ Bn } & RE & - & - & $\begin{array}{l}90.85 \\
(+5.45)\end{array}$ & $\begin{array}{l}90.91 \\
(+2.59)\end{array}$ & $\begin{array}{l}75.68 \\
(+7.83)\end{array}$ & $\begin{array}{l}80.89 \\
(+4.27)\end{array}$ \\
\hline & $\mathrm{ME}$ & - & - & $\begin{array}{l}91.79 \\
(+7.69)\end{array}$ & $\begin{array}{l}90.47 \\
(+2.00)\end{array}$ & $\begin{array}{l}76.94 \\
(+885)\end{array}$ & $\begin{array}{l}81.85 \\
(+199)\end{array}$ \\
\hline \multirow{3}{*}{ Hi } & & 85.97 & 87.27 & & & 72.43 & 79.52 \\
\hline & RE & $(+8.21)$ & $(+3.69)$ & - & - & $(+10.84)$ & $(+0.62)$ \\
\hline & $\mathrm{ME}$ & $\begin{array}{l}85.48 \\
(+7.48)\end{array}$ & $\begin{array}{l}87.21 \\
(+3.04)\end{array}$ & - & - & $\begin{array}{l}75.14 \\
(+13.13)\end{array}$ & $\begin{array}{l}81.00 \\
(+1.36)\end{array}$ \\
\hline \multirow{3}{*}{ Te } & & 85.11 & 83.91 & 86.27 & 88.40 & & \\
\hline & RE & $(+20.47)$ & $(+6.08)$ & $(+19.24)$ & $(+5.47)$ & - & - \\
\hline & $\mathrm{ME}$ & $\begin{array}{l}83.64 \\
(+17.16)\end{array}$ & $\begin{array}{l}84.93 \\
(+7.98)\end{array}$ & $\begin{array}{l}87.36 \\
(+14.67)\end{array}$ & $\begin{array}{l}88.91 \\
(+5.54)\end{array}$ & - & - \\
\hline \multirow{4}{*}{ ALL } & & 87.68 & 89.30 & 91.50 & 92.61 & 76.10 & 8248 \\
\hline & $\mathrm{RE}$ & $(+6.49)$ & $(+2.41)$ & $(+4.28)$ & $(+0.15)$ & $(+7.77)$ & $(+1.76)$ \\
\hline & $\mathrm{ME}$ & 89.83 & 89.99 & 92.74 & 92.90 & 78.75 & 83.96 \\
\hline & & $(+7.43)$ & $(+1.50)$ & $(+5.81)$ & $(+0.98)$ & $(+8.13)$ & $(+1.99)$ \\
\hline
\end{tabular}

Table 16: Accuracy comparison between LMx10 and Ix10 for different source and target languages. The corresponding gains from zeroshot is reported in parentheses. 